\title{
La asociatividad de las Mype regulada en el Decreto Legislativo 1086 y su impacto en el emporio Gamarra $^{1}$
}

The Associativity of the MSER regulated by Legislative Decree 1086 and its Impact on Emporio Gamarra

Sara Ynés Tello Cabello

http://dx.doi.org/10.21503/lex.v10i9.384

\section{Resumen}

El objetivo de la investigación es demostrar las virtudes y el grado de eficacia de la asociatividad de las Mype peruanas, particularmente en el emporio Gamarra, la promoción de mecanismos que permitan el impulso y la evaluación de los criterios que se han venido adoptando en las políticas gubernamentales. Para ello, nos remitiremos a los cuadros estadísticos proporcionados en el riguroso trabajo de investigación elaborado por el Ministerio de Trabajo y de la Promoción del Empleo, titulado Gobiernos locales iniciándose en la promoción de las Mype: necesidades actuales de Gamarra frente al TLC(2008). Estudio en el que se consignan cuadros estadísticos que muestran variables de análisis reveladores que dan cuenta de un perfil más cercano de los empresarios gamarrinos y que develan el grado de probabilidad de implementación de los objetivos propuestos con la nueva Ley Mype.

Palabras clave: Mype, asociatividad de la Mype, emporio Gamarra.

\begin{abstract}
The objective of this research is to demonstrate the virtues and the effectiveness of the associativity of the Peruvian MSEs, particularly in the emporium Gamarra, the promotion of mechanisms for the promotion and evaluation of the criteria have been adopted in the government policies; to do this, we refer to the statistical table provided in the rigorous research work developed by the promotion of MSEs, Gamarra against current needs NAFTA 2008. Study than are recognized statistical table show revealing analysis variables that account

\footnotetext{
Esta tesis puede ser revisada en la biblioteca de la Universidad de Lima. Código de Clasificación: 347.51 T35 (TM) Doctora en educación por la Universidad Alas Peruanas. Profesora de la UAP.
}

2 Abogada por la Universidad Inca Garcilaso de la Vega, Magister en Derecho Empresarial por la Universidad de Lima.
\end{abstract}


for a more close of business of Gamarra and reveal the degree of likelihood of implementation of the objectives with the new law MSEs.

Key words: MSEs, the MSEs Associativity, emporio Gamarra.

\section{Introducción}

La promoción de la asociatividad de las Mype en el Perú, por intermedio del Decreto Legislativo No 1086, Ley de Promoción de la Competitividad, Formalización y Desarrollo de la Micro y Pequeña Empresa y del Acceso al Empleo Decente, Ley Mype, promulgado en septiembre de 2008, representa una de las tareas de mayor trascendencia de la nueva legislación Mype, puesto que tiene como objetivo implementar medidas destinadas a consolidar a este grupo empresarial, convertido desde hace dos décadas en el motor del desarrollo empresarial local. Grupo emprendedor constituido en sus inicios por migrantes provincianos que se vieron compelidos a operar de espaldas al mercado formal ante la falta de oportunidades promovidas por los gobiernos de turno; no obstante ello, en la actualidad las pequeñas y medianas empresas vienen adquiriendo un nuevo rostro y dinámica gracias al compromiso del Estado y el empuje de sus propulsores, y requieren ser evaluadas convenientemente para efectos de su integración al sistema formal y posterior potenciación dentro del mercado interno y externo. Cabe advertir que el compromiso del Gobierno peruano para la promoción de la asociatividad empresarial en las Mype ha sido puesto de manifiesto por intermedio de su política de inserción en la economía global, canalizada a través de la negociación de Tratados de Libre Comercio con los Estados Unidos de Norteamérica, China, Tailandia, Singapur, Canadá, entre otros. Adopción de medidas que, sin embargo, todavía no ha logrado difundirse adecuadamente entre sus actores ni se han elaborado estrategias consensuadas a nivel gubernamental; así como no se ha conseguido revertir determinados factores culturales, atribuidos erróneamente a este sector empresarial, como probables causantes de la falta de asociatividad alcanzada; además de otras falacias, tales como su poco grado de educación, poco interés para la capacitación, resistencia a integrarse al mercado formal, entre otras que, gracias a los últimos estudios que se vienen efectuando, van perdiendo peso. La política de apertura comercial peruana referida, sin embargo, genera nuevos retos y posibilidades. Una de ellas es la posibilidad de incrementar las exportaciones. Sobre este particular, habría que señalar que, aunque el nivel de exportaciones alcanzados por las Mype se viene incrementando favorablemente, todavía no alcanza el volumen proyectado por los especialistas. Así, en el debate del tema central de CADE 2008, "La reforma del sector privado", se propuso tomar medidas para "acelerar la formalización de las Mype, su asociatividad entre ellas y con medianas y grandes empresas nacionales, con miras a incrementar su participación en el comercio exterior. (...) de tal manera que el 2,5\% 
(5760) del total de empresas nacionales que exportaron durante el 2007, se incremente" (Gestión, 2008). Pese a ello, debe recordarse que el potencial exportador de las Mype podría extenderse a un espectro mucho más amplio, puesto que de un universo peruano de " 2,52 millones de empresas, el $91,7 \%$ son microempresas (menos de diez trabajadores), 6,9\% son pequeñas y el 1,4\% (35252) son grandes empresas, tal como lo refiere el Consejo Nacional para el Desarrollo (Cesden)" (Gestión, 2008b). Se debe también resaltar la importancia de las microempresas en el ámbito laboral local, cuya incidencia abarca a "cerca de ocho millones de personas y que contribuyen al PBI en aproximadamente el 28\% del total (Gestión, 2008c). Asimismo, se precisa de herramientas comerciales que rompan con enfoques tradicionales contrarios al trabajo en equipo y el emprendimiento de acciones que vayan más allá de su normativa, tal como lo recomendara el estudio efectuado por PROEXPANSIÓN para PROMPYME el año 2003. Adecuación que resultará decisiva para su supervivencia dentro de las exigencias de la economía mundial. Por ello, algunos han afirmado en tono ciertamente desolador que "la pequeña empresa de Gamarra aislada no tiene absolutamente nada que hacer, no tiene la menor chance de sobrevivir. Para que las empresas puedan competir en el mercado globalizado tendrían que modernizarse y crecer de manera espectacular, y este proceso duraría por lo menos 5 años; para entonces ya todas estarían muertas. La única manera de sobrevivir, en el corto plazo, es crecer horizontalmente mediante la cooperación empresarial e institucional" (Villarán, 1998). Por ello, consideramos importante abordar la problemática de la asociatividad empresarial para intentar hallar las causas de su poca recurrencia y proponer alternativas que hagan propicio el desarrollo y competitividad de las Mype, previstas en el Decreto Legislativo No 1086. Consideraciones por las cuales nuestro trabajo de investigación asume la difícil tarea de encontrar una respuesta al aparente desinterés por la asociatividad de los empresarios Mype, delimitando su ámbito de estudio al emporio comercial textil de Gamarra.

\section{Objetivo general}

Nuestro trabajo de investigación tiene como objetivo general:

1. Demostrar el grado de eficacia de la asociatividad en las Mype peruanas, particularmente en el emporio Gamarra.

\section{Objetivos especificos}

El objetivo específico de la investigación consiste en:

1. Determinar los mecanismos de promoción que permiten el impulso de las Mype.

2. La evaluación de los criterios que se vienen adoptando a través de las políticas gubernamentales. 
3. Determinar la situación actual de la capacitación y asistencia técnica recibida en las Mype de Gamarra.

Hipótesis general

La ineficacia en la promoción de la asociatividad en las Mype en el emporio Gamarra probablemente se debería a factores de orden normativo, cultural y de políticas gubernamentales deficientes.

La hipótesis de nuestro estudio está compuesta por tres variables:

i) Variable normativa: $\mathrm{x}$

ii) Variable políticas gubernamentales: $y$

iii) Variable factor cultural: $\mathrm{z}$

\section{Conceptos importantes}

Asociatividad empresarial. Es una estrategia para enfrentar a los mercados globalizados. Las Mype, sin perjuicio de las formas societarias previstas en las leyes sobre la materia, pueden asociarse para tener un mayor acceso al mercado privado y a las compras estatales. Todos los beneficios y medidas de promoción para que las Mype participen en las compras estatales incluyen a los consorcios que sean establecidos entre las Mype.

Compras del Estado. Las Mype participan en las contrataciones del Estado de acuerdo con la normativa vigente. Las entidades del Estado deberán programar no menos del $40 \%$ de sus contrataciones para que sean atendidas por las Mype. En las contrataciones de bienes y servicios, las entidades del Estado prefieren a las Mype cuando cumplen con las especificaciones técnicas establecidas en las bases de los procesos de contratación y oferten en condiciones similares de calidad, oportunidad y precio. Igualmente, se dará preferencia a las Mype de la región o localidad del lugar donde se realicen las compras estatales, respecto de los bienes y servicios que puedan ser suministrados por las Mype regionales o locales, siempre que cumplan con las especificaciones técnicas establecidas en las bases y oferten en condiciones similares de calidad, oportunidad y precio. Como sistema alternativo a la obligación de la garantía de fiel cumplimiento, en caso de suministros periódicos de bienes o prestación de servicios de ejecución periódica, así como en los contratos de consultoría y ejecución de obras, las Mype podrán optar porque por concepto de dicha garantía la entidad retenga el 10\% del monto del contrato original. Para estos efectos, la retención de dicho monto se efectuará durante la primera mitad del número total de pagos a realizarse, en forma prorrateada en cada pago, con cargo de ser devuelto a la finalización del mismo. 
Clúster. Conjunto de empresas que se encuentran integradas a un mercado que comparten relaciones de conocimientos e insumos. Esta aglomeración productiva genera economías de escala y efectos de desbordamiento que hace que los costos medios de producción de las empresas inmersas en el clúster se reduzcan en el tiempo.

Emporio Gamarra. El emporio gamarrino se encuentra asentado en el distrito de La Victoria. Dentro de su espectro, según el estudio elaborado por el Instituto Nacional de Estadística e Información (INEI), sobre la base de datos de Actualización de Negocios y el III Censo Económico, el área central de Lima contaba en 1996 con 67943 establecimientos con un crecimiento de 31740 en los últimos tres años, y de todas ellas el 96,7\% eran PYME. Respecto a la densidad de establecimientos, el distrito de La Victoria concentraba el 21,1\% de dichos establecimientos. Las actividades que predominan en Gamarra son las de confecciones y de vulcanizado, asociados a los servicios de mantenimiento de vehículos. Sobre este particular, resulta fundamental remitirnos a la fuente del MTPE que concluye que "La Victoria concentraba, el año 2007, el 54,8\% de los establecimientos de confecciones y el $34 \%$ de los establecimientos vinculados a servicios de mantenimiento de vehículos de todo el país"(Villarán, 1998). Pese a que en La Victoria se concentran actividades económicas y de servicios adicionales de trascendencia no solo limeña sino dentro del espectro nacional, es en el sector de confecciones donde su prevalencia es más que notoria. Precisamente, de acuerdo a los resultados estadísticos efectuados hace pocos años, "Gamarra concentra aproximadamente 15 mil unidades económicas de producción, comercio y servicios, lo cual significa el 45,37\% de los talleres de costura y el 37,84\% de las boutique de Lima Metropolitana. Y, en pleno proceso de modernización y crecimiento de los supermercados y tiendas por departamentos, este emporio comercial y productivo de confecciones goza del $57 \%$ de preferencia de los consumidores de este segmento"(Villarán, 1998). No obstante ello, en un estudio efectuado por la encuestadora de la Universidad de Lima, a fines de 2009, se determinó que el porcentaje de limeños que prefiere ir de compras a Gamarra es de 74,9\%, mientras que el 16,1\% opta por ir a comprar en Saga y Ripley. Asimismo, se menciona que, a noviembre de 2009, cuenta con más de 17000 confeccionistas, y con una afluencia de 150 mil personas cada día (estas cifras suelen duplicarse en Fiestas Patrias y durante las celebraciones navideñas) (Perú21, 2009).

Empresa. Unidad económica generadora de rentas de tercera categoría conforme a la ley del Impuesto a la Renta, con una finalidad lucrativa.

Instrumento de promoción. Mecanismo que promueve el Estado para facilitar el acceso de las Mype a los servicios empresariales y a los nuevos emprendimientos, con el fin de crear un entorno favorable a su competitividad, promoviendo la conformación de mercados de servicios financieros y no financieros, de calidad, descentralizado y pertinente a las necesidades y potencialidades de dichos estratos empresariales. 
Micro y Pequeña Empresa. Es la unidad económica constituida por una persona natural o jurídica, bajo cualquier forma de organización o gestión empresarial contemplada en la legislación vigente, que tiene como objeto desarrollar actividades de extracción, transformación, producción, comercialización de bienes o prestación de servicios.

Politica estatal. El Estado promueve un entorno favorable para la creación, formalización, desarrollo y competitividad de las Mype y el apoyo a los nuevos emprendimientos, a través de los Gobiernos Nacionales, Regionales y Locales; y establece una marco legal e incentiva la inversión privada, generando o promoviendo una oferta de servicios empresariales destinados a mejorar los niveles de organización, administración, tecnificación y articulación productiva y comercial de la Mype, estableciendo políticas que permitan la organización y asociación empresarial para el crecimiento económico con empleo sostenible.

Registro de la Micro y Pequeña Empresa. Para acceder a los beneficios de la Ley Mype se deberá tener el certificado de inscripción o de reinscripción vigente en el REMYPE.

\section{Metodología}

\section{Tipo de investigación}

Por el tipo de investigación, el presente estudio reúne las condiciones necesarias para ser denominado como investigación aplicada, pues busca establecer una serie de proposiciones coherentes sobre el fenómeno de estudio.

\section{Nivel de Investigación}

Asimismo, la investigación es de un nivel correlacional.

Es explicativo porque se analizará la ratio essendi de la Mype regulado en el Decreto Legislativo 1086; con la finalidad de presentar, detallar, interpretar y explicar lo referente a esta figura, presentando las principales manifestaciones de este (naturaleza actual y estructura). En efecto, describiremos los aspectos conceptuales de esta figura, su presencia en otras legislaciones, su tratamiento en el sistema jurídico peruano y la problemática que este presenta en nuestro país.

\section{Diseño de investigación}

Se ha indicado que esta investigación es no experimental de corte transversal, con lo cual podremos establecer y probar el grado de resistencia a la figura de la asociatividad empresarial de los empresarios de las Mype de Gamarra. Estas variables de estudio son: a) variable de factor normativo, b) variable de políticas gubernamentales, y c) variable de factor cultural. 
El diseño específico en la verificación de la hipótesis estará orientado por el siguiente esquema:

$\mathrm{O}_{\mathrm{X}} \quad \mathrm{M}$, es la muestra representativa

$\mathrm{Mr} \mathrm{O}_{\mathrm{X}}$, observaciones de la variable "X"

$\mathrm{O}_{\mathrm{y}} \mathrm{O}_{\mathrm{y}}$, observaciones de las variable " $\mathrm{y}$ "

r, nivel de correlación entre $\mathrm{O}_{\mathrm{x}}$ y $\mathrm{O}_{\mathrm{y}}$

La hipótesis se comprobará utilizando el presente diseño de investigación, midiendo el nivel de correlación existente entre las variables " $x$ " y " $y$ ", recolectando datos. Luego de ello podremos saber cómo se puede comportar la variable " $y$ ” en función de la variable " $x$ ".

\section{Métodos de investigación}

Los métodos científicos empleados en la investigación son:

- Análisis-Sintesis. A través de este método se descompondrá en parte la figura de la Mype, para estudiar las situaciones, efectos y disfuncionalidades en el sistema normativo que la misma pudiese presentar.

- Descriptivo-Explicativo. Mediante este método se acopiará toda la información referida a las Mype, a fin de explicar a cabalidad esta figura.

- Inductivo-Deductivo. A través del método inductivo, se partirá de las situaciones particulares o específicas de las Mype, a fin de llegar a lo general de esa figura. Asimismo, con el método deductivo, se seguirá el procedimiento inverso.

- Comparativo. Mediante este método se confrontará y comparará el tratamiento de la Mype con relación a otros sistemas jurídicos, a fin de llegar a comprender las características peculiares de esta figura.

Técnicas e instrumentos de recolección de datos

Técnicas. Las técnicas para la recolección de datos fueron la observación, posteriormente se recurrió al análisis documental, para finalmente recolectar los datos de los objetos de estudio a través de la encuesta. La información se recopiló tomando como unidades de análisis a cada micro y pequeño empresario del emporio Gamarra.

Instrumentos. Los instrumentos aplicados para el recojo de datos son correspondientes a cada técnica seleccionada, datos recogidos por observación de las unidades. El análisis se 
ha hecho a través de una guía de observación. También se construyó una guía de análisis documental para organizar los registros de información oficial sobre las Mype y se elaboró un cuestionario tipo Lickert.

\section{Tablas y análisis de datos}

Para el procesamiento de los datos, se los tabuló y organizó en cuadros unidimensionales y bidimensionales para el análisis de las variables con sus respectivas interpretaciones, para posteriormente establecer la relación entre ellas mediante las técnicas estadísticas no paramétricas. El parámetro a utilizar, de acuerdo a la naturaleza de las variables, fue el Coeficiente de Correlación de Rango de Spearman, que determinará el grado de asociación o relación de una variable sobre la otra, considerando para ello un nivel de significancia de 0,05.

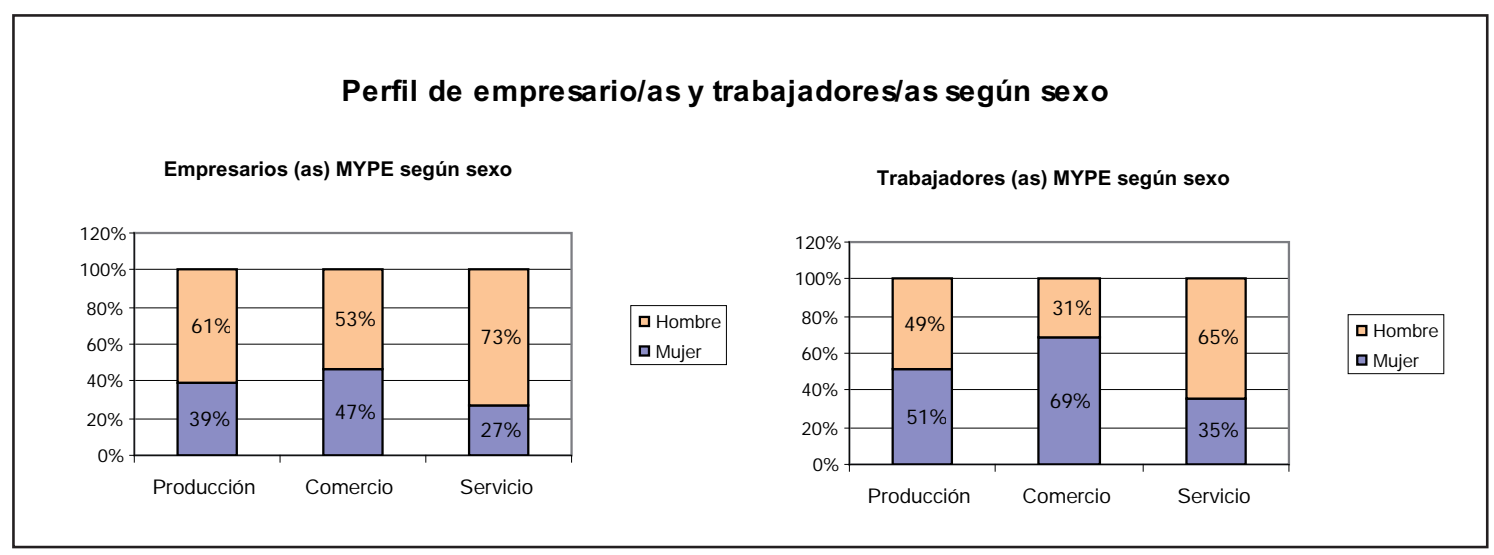

Fuente: Ministerio de Trabajo y Promoción del empleo, año 2008.

\section{Figura 1}

De las estadísticas, se puede observar una diferenciación marcada en cuanto a las preferencias de los talleres de producción y servicios de Gamarra. Lo cual no deja de ser importante en la medida que nos permite determinar la composición de género en cada una de las actividades referidas. Composición que, a su vez, permitirá contar con elementos informativos que posibiliten resultados positivos en la tarea de implantar estrategias que potencien lo avanzado por las Mype puestas en evaluación en el presente trabajo, así como en la elaboración de estrategias de capacitación y consolidación de este sector empresarial. Entendimiento que resulta fundamental en las políticas gubernamentales que se pretendan implementar para el fomento de la asociatividad de las Mype de Gamarra, entre otras medidas. 
Grado de Instrucción de los Empresarios/as: 169 Grado de Instrucción de los trabajadores/as: 222

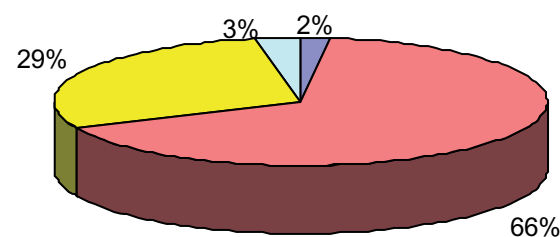

$\square$ Primaria $\square$ Secundaria $\square$ Superior $\square$ No sabe $\square$ No opina

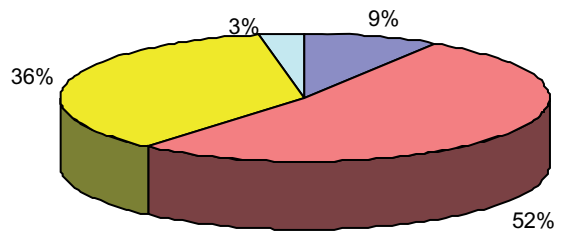

$\square$ Primaria $\square$ Secundaria $\square$ Superior $\square$ No sabe $\square$ No opina

Fuente: Ministerio de Trabajo y Promoción del empleo, año 2008

Figura 2

La Figura 2 que arroja el estudio del MTPE sobre Gamarra se refiere al grado de educación de los integrantes de este emporio comercial. El estudio revela que en cuanto al grado de instrucción de quienes se encargan de dirigir la gestión de las Mype, "el 52\% tiene educación secundaria, y superior el 36\%. Mientras que entre las trabajadoras y los trabajadores esta cifra asciende al 95\%: con secundaria el 66\% y con superior el 29\%" ${ }^{3}$. Lo relevante de la muestra sobre el grado de instrucción de los empresarios y empleados de Gamarra es, sin duda, el hecho de romper una vieja creencia de que quienes integran este emporio comercial no cuentan con una educación mediana como para poder ser insertados convenientemente en el mercado formal ni encontrar respuesta en las medidas gubernamentales, por la creencia de que manejan códigos totalmente diferentes al utilizado por el sector integrado al sistema económico oficial. Contrariando esa vieja perspectiva, el estudio del MTPE no deja de ser alentador, además de irradiar una luz de optimismo en el éxito de los programas que se vayan a implementar para potenciar este conglomerado comercial, si convenimos en que la educación de sus integrantes constituye un pilar decisivo para su éxito. Por tanto, esperemos que los resultados de esta muestra se lleguen a difundir, en cuanto sea necesario, por las instituciones que vienen haciéndose cargo de promover la consolidación de las Mype en el Perú. En tal sentido, consideramos fundamental que el Ministerio de la Producción, el MTPE, los Gobiernos Regionales y Locales, a través de los organismos pertinentes, puedan difundir en los ciudadanos esta muestra, de tal manera que se vaya borrando el estereotipo aquel que sindica a este sector pujante como uno integrado por gente sin instrucción, dueño de una serie de estereotipos y totalmente divorciado del ámbito oficial.

MINISTERIO DE TRABAJO Y PROMOCIÓN DEL EMPLEO (MTPE). Gobiernos locales iniciándose en la promoción de las Mype: necesidades actuales de Gamarra frente al TLC. En: www.mintragob.pe. Lima, 2008, p.100. 


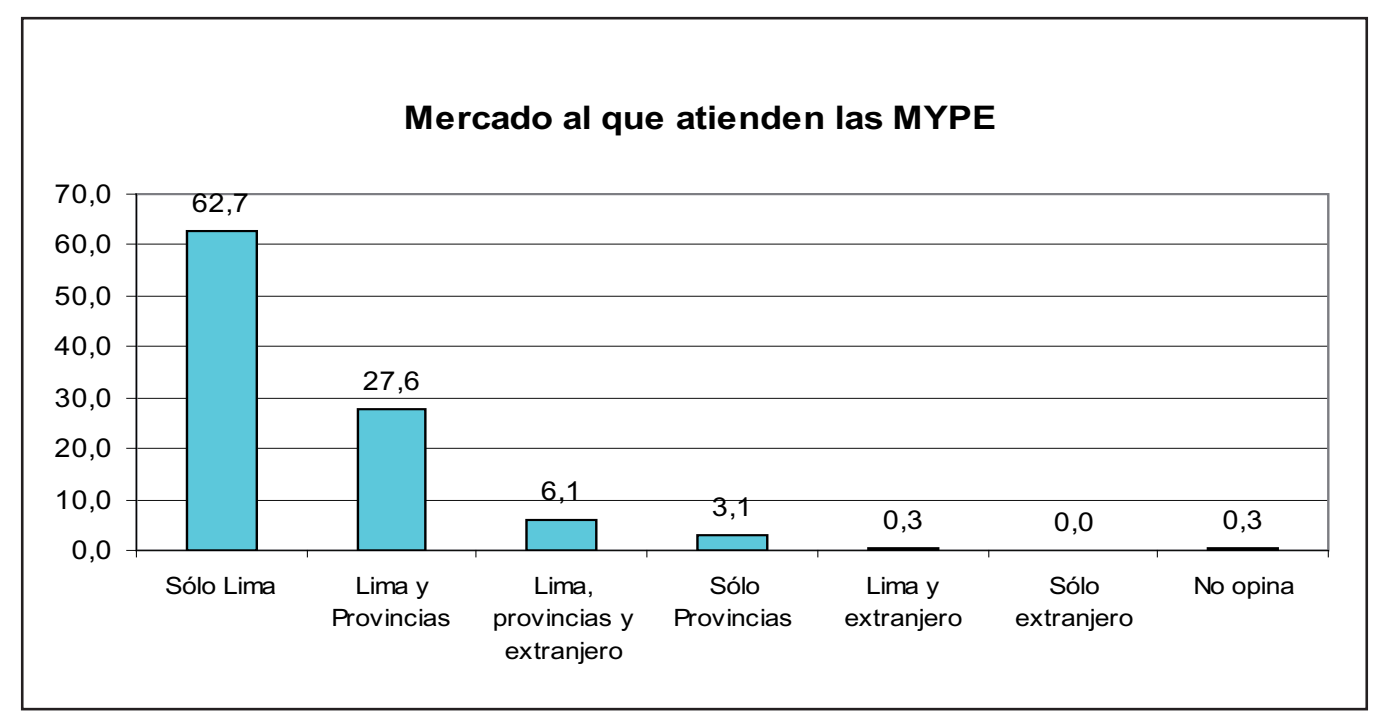

Fuente: Ministerio de Trabajo y Promoción del empleo, año 2008.

Figura 3

Como tercer punto, el estudio elaborado por el MTPE proporciona, además, otra información valiosa vinculada al rango de acción del mercado gamarrino. Al respecto, se arriba a la conclusión de que "el principal mercado de las Mype entrevistadas es Lima, donde el $62,7 \%$ atiende exclusivamente este mercado; el 27,6\% restante atiende a provincias, y el $6,1 \%$ ha logrado ampliar su mercado al extranjero (están exportando de manera directa o indirecta)". ${ }^{4}$ Consideramos que esta muestra debe ser evaluada con mucho detenimiento puesto que nos permite apreciar el grado de incursión del emporio Gamarra en el mercado local, nacional y a nivel externo. Así las cosas, se observa que las Mype gamarrinas tienen como mercado principal a la capital, lo cual no deja de ser interesante, dado que ello implica una dura competencia con las cadenas de tiendas como Ripley, Saga Falabella, entre otras firmas poderosas que vienen posicionándose agresivamente en el mercado interno.

4 MINISTERIO DE TRABAJO Y PROMOCIÓN DEL EMPLEO (MTPE). Gobiernos locales iniciándose en la promoción de las Mype: necesidades actuales de Gamarra frente al TLC. En: www.mintragob.pe. Lima, 2008, p.111. 
Tabla 1. Capacitación recibida por las Mype, por tipo de Mype, según sexo y función dentro de la empresa

\begin{tabular}{|c|c|c|c|c|c|}
\hline & \multirow{2}{*}{$\begin{array}{c}\text { No de capacitaciones } \\
\text { recibidas }\end{array}$} & \multicolumn{3}{|c|}{ Tipo principal de Mype } & \multirow[t]{2}{*}{ Total } \\
\hline & & Producción & Comercio & Servicio & \\
\hline \multirow{6}{*}{ Empresaria } & 0 & 83,8 & 85,7 & 70,8 & 80,9 \\
\hline & 1 & 16,2 & 3,6 & 8,3 & 10,1 \\
\hline & 3 & & 7,1 & 8,3 & 4,5 \\
\hline & 2 & & 3,6 & 4,2 & 2,2 \\
\hline & 5 & & & 4,2 & 1,1 \\
\hline & 10 & & & 4,2 & 1,1 \\
\hline & Total & 100,0 & 100,0 & 100,0 & 100,0 \\
\hline \multirow{8}{*}{ Empresario } & 0 & 80,7 & 93,8 & 81,3 & 83,7 \\
\hline & 1 & 8,8 & 3,1 & 14,1 & 9,8 \\
\hline & 3 & 1,8 & 3,1 & 1,6 & 2,0 \\
\hline & 6 & 3,5 & & 1,6 & 2,0 \\
\hline & 2 & 3,5 & & & 1,3 \\
\hline & 4 & 1,8 & & & 0,7 \\
\hline & 5 & & & & 0,7 \\
\hline & Total & 100,0 & 100,0 & 100,0 & 100,0 \\
\hline
\end{tabular}

\begin{tabular}{|c|c|c|c|c|c|}
\hline & \multirow{2}{*}{$\begin{array}{c}\text { No de capacitaciones } \\
\text { recibidas }\end{array}$} & \multicolumn{3}{|c|}{ Tipo principal de Mype } & \multirow[t]{2}{*}{ Total } \\
\hline & & Producción & Comercio & Servicio & \\
\hline \multirow{8}{*}{ Trabajadora } & 0 & 83,3 & 80,5 & 86,7 & 82,5 \\
\hline & 1 & 12,5 & 9,8 & & 8,8 \\
\hline & 2 & & 2,4 & 13,3 & 3,8 \\
\hline & 3 & & 2,4 & & 1,3 \\
\hline & 5 & & 2,4 & & 1,3 \\
\hline & 7 & 4,2 & & & 1,3 \\
\hline & 15 & & 2,4 & & 1,3 \\
\hline & Total & 100,0 & 100,0 & 100,0 & 100,0 \\
\hline \multirow{8}{*}{ Trabajador } & 0 & 82,6 & 88,9 & 92,9 & 88,4 \\
\hline & 1 & 4,3 & & 7,1 & 4,3 \\
\hline & 2 & 4,3 & & & 1,4 \\
\hline & 4 & 4,3 & & & 1,4 \\
\hline & 6 & 4,3 & & & 1,4 \\
\hline & 9 & & 5,6 & & 1,4 \\
\hline & 12 & & 5,6 & & 1,4 \\
\hline & Total & 100,0 & 100,0 & 100,0 & 100,0 \\
\hline
\end{tabular}


Tabla 2. Asistencia técnica recibida por las Mype por tipo de Mype, según sexo y función dentro de la empresa

\begin{tabular}{|c|c|c|c|c|}
\hline \multirow[t]{2}{*}{ Sexo } & \multirow{2}{*}{$\begin{array}{l}\text { Número de asistencias } \\
\text { técnicas recibidas }\end{array}$} & \multicolumn{2}{|c|}{ Tipo principal de Mype } & \multirow[b]{2}{*}{ Total } \\
\hline & & Producción & Servicio & \\
\hline \multirow{3}{*}{ Empresaria } & 0 & 91,9 & 87,5 & 90,2 \\
\hline & 1 & 5,4 & 8,3 & 6,6 \\
\hline & 4 & 2,7 & 4,2 & 3,3 \\
\hline & Total & 100,0 & 100,0 & 100,0 \\
\hline \multirow{4}{*}{ Empresario } & 0 & 94,7 & 85,9 & 90,1 \\
\hline & 1 & 5,3 & 12,5 & 9,1 \\
\hline & 2 & & 1,6 & 0,8 \\
\hline & Total & 100,0 & 100,0 & 100,0 \\
\hline \multirow{5}{*}{ Trabajadora } & 0 & 91,7 & 86,7 & 89,7 \\
\hline & 1 & 8,3 & & 5,1 \\
\hline & 2 & & 6,7 & 2,6 \\
\hline & 5 & & 6,7 & 2,6 \\
\hline & Total & 100,0 & 100,0 & 100,0 \\
\hline \multirow{5}{*}{ Trabajador } & 0 & 87,0 & 85,7 & 86,3 \\
\hline & 1 & 4,3 & 7,1 & 5,9 \\
\hline & 2 & 8,7 & & 3,9 \\
\hline & 3 & & 7,1 & 3,9 \\
\hline & Total & 100,0 & 100,0 & 100,0 \\
\hline
\end{tabular}

Los resultados obtenidos revelan, sin duda, una información interesante en la medida que, por lo general, solo se le asigna a las universidades y centros de estudio la competencia idónea para esta tarea. No obstante ello, el estudio del MTPE hace notar que este tipo de percepción no es del todo correcto y que debería tenerse presente que el 41,7\% de Mype de servicio prefieren ser capacitadas por la gran empresa. Esto último tal vez en razón de que prefieren recibir pautas de aquellos que se ejercitan día a día en labores similares a los empresarios Mype, solo que en ámbitos de acción mayores. Sin dejar de contar con cierta resistencia a lo puramente conceptual o teórico, rasgo con el cual se suele vincular a los centros de educación superior en el Perú. 


\section{Conclusiones}

1. Como primer punto habría que especificar que para demostrar la eficacia de la asociatividad de las Mype tendríamos que analizar la eficacia normativa y la relevancia que puede tener el contenido normativo en el logro de los objetivos trazados por el legislador, en este caso a través del Decreto Legislativo No 1086, Ley de Promoción de la Competitividad, Formalización y Desarrollo de la Micro y Pequeña Empresa y del Acceso al Empleo Decente, Ley Mype, prevista para la promoción y mejora de la asociatividad en las Mype de Gamarra. Arribamos a la conclusión de que la variación normativa con la Ley No 28015 , Ley Mype anterior, no es sustancial ni determinante en la obtención de las metas planteadas. Aun cuando en la normativa vigente se han añadido algunos beneficios para las Mype que no estuvieron presentes en la ley anterior, tales como los beneficios laborales y tributarios, creemos que los problemas de implementación y la poca acogida que se obtuvo en los actores comprendidos en este sector, se debe a cuestiones que tienen que ver con la gestión e implementación inadecuada de la norma. En tal sentido, queda demostrado que no existe la ineficacia de la asociatividad en las Mype gamarrinas, al no existir programas de implementación de dicha legislación ya que no fueron lo suficientemente bien elaborados ni existió el compromiso requerido por parte de los gobiernos de turno para hacer que este plan funcione a cabalidad.

2. La falta de los mecanismos de promoción no permite el impulso de la Mype, toda vez que aún no se ha implementado el acceso a los programas y medidas de fomento al desarrollo empresarial, que debería estar articulado para priorizar aquellas empresas que se agrupen en unidades asociativas o clúster o se inserten en procesos de subcontratación o cadenas productivas de exportación, mecanismo de promoción que favorecería al empresario gamarrino. Se cree aún que el empresario gamarrino no se encuentra preparado para la ejecución de los planes de gobierno por su escasa preparación académica, situación que no es real. De acuerdo con el estudio del MTPE, que nos sirve de insumo para el presente trabajo de investigación, esa idea sesgada de que el empresario gamarrino no logra llegar a los mecanismos de promoción es falsa. Creemos que se debe añadir un mayor dinamismo a la tarea de promoción señalada, puesto que este grupo de emprendedores requiere del apoyo del Gobierno, ahora que cuenta con un espacio ganado por su propio esfuerzo e iniciativa. Por tanto, el Estado debe priorizar este objetivo otorgando las partidas presupuestarias necesarias, así como por intermedio de la creación de programas técnicos que respondan a las necesidades reales de estos actores, y sobre todo que estos programas se sostengan en el tiempo hasta la obtención de las metas planteadas al momento de su promulgación.

3. La evaluación de los criterios que se viene adoptando a través de las políticas gubernamentales no están siendo del todo claras. En la participación del Estado, con respecto a la 
gestión gubernamental, es donde se encuentra gran parte de la problemática Mype. Sobre la asociatividad en particular, habría que señalar que no se encontraron los mecanismos ni estrategias que surtieran efecto en la tarea de internalizar esta práctica comercial en los empresarios gamarrinos. El esfuerzo del Gobierno de concretar acuerdos comerciales con otros países, así como la serie de beneficios en las compras estatales, entre otros, no fueron suficiente para potenciar esta herramienta. El estudio elaborado por el MTPE proporciona, además, otra información valiosa vinculada al rango de acción del mercado gamarrino. $\mathrm{Al}$ respecto, se arriba a la conclusión de que "el principal mercado de las Mype entrevistadas es Lima, el 62,7\% atiende exclusivamente este mercado; el 27,6\% restante atiende a provincias, y el 6,1\% ha logrado ampliar su mercado al extranjero (están exportando de manera directa o indirecta)" (MTPE, 2008). Consideramos que esta muestra debe ser evaluada con mucho detenimiento, puesto que nos permite apreciar el grado de incursión del emporio Gamarra en el mercado local, nacional y a nivel externo. Así las cosas, se observa que las Mype gamarrinas tienen como mercado principal a la capital, lo cual no deja de ser interesante dado que ello implica una dura competencia con las cadenas de tiendas como Ripley, Saga Falabella, entre otras firmas poderosas que vienen posicionándose agresivamente en el mercado interno. Pese a las preferencias internas de los productos producidos en Gamarra, es interesante hacer notar el bajo nivel de exportación que se viene efectuando en este rubro (6,1\% del total de producción). Resultado que requiere una pronta reingeniería en las estrategias que se vienen aplicando en la actualidad para la obtención de los beneficios derivados de los acuerdos de complementación económica celebrados por nuestro país con sus recientes socios comerciales. Por tanto, esta tarea constituye una prioridad en la agenda gubernamental para efectos de cristalizar los objetivos del Gobierno a través de la Ley Mype.

4. Pese a la importancia de la capacitación y la asistencia técnica en las Mype para su desarrollo, no logra concretarse aun en nuestro país; con respecto a la situación actual de la capacitación recibida en las Mype, el estudio del MTPE arriba a la conclusión de que, "solo ha recibido capacitación el 19\% de empresarias, el 16,3\% de empresarios, el 17,5\% de trabajadoras y el $11,6 \%$ de trabajadores. Es decir, hay una ausencia significativa de capacitación en las empresas". Cuadro que revela una mejor disposición a la capacitación de parte de las mujeres dedicadas al servicio que operan en el emporio victoriano. Esta afirmación se fundamenta en que "entre las mujeres empresarias quienes han recibido mayor número de capacitaciones son las empresas de servicio, alcanzando un 29\%, difiriendo notablemente con las empresas de producción y comercio, cuyas empresarias que han recibido capacitación constituyen el 16,2\% y 14,3\%, respectivamente" (MTPE, 2008). Pese al porcentaje referido, creemos que este grupo femenino puede mejorar su grado de capacitación. Creemos que si las cifras no han dado el salto decisivo es por cuestiones de 
falta de apoyo institucional que cristalice el sentir de estas integrantes del conglomerado victoriano. Asimismo, el tema de la capacitación en las Mype de Gamarra muestra un dato que causa preocupación: los encuestados afirmaron que en las Mype de producción, el total de empresarias que dicen haber recibido capacitación solo la tuvieron una vez; mientras que en comercio se llegaron a tres capacitaciones, y lo mismo sucedió en las Mype de servicios. La preocupación se justifica si reparamos en que con solo una capacitación o dos, no se puede decir que ya se logró el objetivo. Esto porque, como bien sabemos, la capacitación debe ser constante dado que el mercado es cambiante y la economía muestra rostros que mutan permanentemente en el tiempo. Por tanto, se debe efectuar un trabajo de docencia para hacer entender esta premisa y crear las condiciones para que se lleguen a concretar tales objetivos. Aunado a ello, de acuerdo con los resultados arribados con el trabajo del MTPE, "la demanda de servicios de capacitación de las empresarias de las Mype de producción, comercio y servicios en Gamarra, prioriza en un 51,7\% las ventas, administración y marketing, como temas de gestión". Esta tendencia debido a que el éxito de las ventas son determinantes en la vida de cualquier empresa, así como la necesidad de contar con una correcta administración y marketing que conlleve a sostener una situación favorable dentro del mercado. De otro lado, proporciona elementos de juicio para sostener que este grupo humano está predispuesto a integrarse al sistema formal y demuestra que sus expectativas trascienden las fronteras internas. Indicador que evidencia una tendencia que rompe con el mito aquel que asigna a este sector un desinterés marcado por integrarse al mercado formal y su reticencia a encontrar vías que le permitan crecer tanto o más que cualquier otra. 


\section{Recomendaciones}

1. Para que la asociatividad de las Mype gamarrinas funcione eficazmente debe existir una implementación en la aplicación normativa de las mismas, es decir, el Estado debe ejecutar políticas, acciones y presupuesto para las Mype, en función de los objetivos trazados por la norma, específicamente asociándose entre ellas a fin de afrontar el mercado producto de la globalización. Debemos tener presente que, de acuerdo con el estudio del MTPE que nos sirve de insumo para el presente trabajo de investigación, dentro del emporio victoriano "existe una relación más pareja entre el número de mujeres y hombres que trabajan en talleres de producción y servicios. Del total de empresarias, el $47 \%$ de ellas está en el comercio de confecciones". En tanto que "las empresas de servicios son eminentemente masculinas, tanto a nivel de propiedad ( $73 \%$ de estas son conducidas por varones) como en el mercado laboral (65\% de trabajadores son hombres)" (MTPE, 2008). De este universo, el 37\% de empresarios y trabajadores provienen de Lima. Entendimiento que resulta fundamental en las políticas gubernamentales que se pretendan implementar para el fomento de la asociatividad de las Mype de Gamarra, entre otras medidas (ver Figura 1).

2. Recomendamos diseñar programas de capacitación tanto para los empresarios como para los empleados. Cursos que bien podrían dictarse los domingos y con capacitadores provenientes de las grandes empresas como de las universidades. Asimismo, se debe tener presente que dentro de este universo existe un gran porcentaje de mujeres con necesidades particulares. Las capacitaciones deben comprender aspectos tales como mejora de la gestión, acceso a la información, mejorar habilidades técnicas, y cursos básicos de comercio internacional y de finanzas, entre otros, con el objeto de hacer notar la necesidad de acoger a la asociatividad empresarial como alternativa de desarrollo y competitividad empresarial; y dejar de lado ese prejuicio obsoleto de que los empresarios gamarrinos no cuentan con preparación para entender los cursos de capacitación programados para el impulso y desarrollo de las Mype. (Ver Figura 2.)

3. Recomendamos que el Estado continúe en su política gubernamental, con la apertura comercial peruana referida, a fin de generar nuevos retos y posibilidades para el micro y pequeño empresario gamarrino. Sin lugar a dudas lo más importante sería incrementar las exportaciones. Se necesita ampliar el mercado Mype de Gamarra. No es conveniente que sus ventas sean de gran volumen solo en Lima (62,7 \%); es necesario que se incrementen las ventas al interior, potenciar el 6,1\% de ventas al exterior y copar el $40 \%$ de ventas al Estado. (Ver Figura 3.) 
4. Es importante que el Estado ofrezca una atención personalizada del servicio, ya que tanto la capacitación y la asistencia técnica deben tener como objetivo resolver problemas específicos identificados en cada unidad productiva, ya sea de comercio o de servicio. La capacitación y la asistencia técnica no debe ser globales, sino individuales, identificando los problemas de gestión, tratando de dar una asistencia técnica más directa al empresario gamarrino. No solo en Gamarra se vende polos. Debe pues existir una capacitación exclusiva sobre bienes y servicios que se otorgan en el emporio Gamarra. (Ver Tablas 1 y 2.)

\section{Referencias}

- Andriani, C. (2003). El nuevo sistema de gestión para las PYMES: un reto para las empresas latinoamericanas. Colombia: Grupo Editorial Norma.

- Cosmópolis, M. (2008). Gobiernos locales iniciándose en la promoción de las Mype: necesidades actuales de Gamarra frente al TLC. Lima: Ministerio de Trabajo y Promoción del Empleo. En: www.mintra.gob.pe

- Dirección Nacional de la Micro y Pequeña Empresa. Actualización de Estadísticas de la Micro y Pequeña Empresa. En: www.mintra.gob.pe. Lima, diciembre de 2007.

- Diario Gestión (2008, octubre 30). Suplemento Gestión en la CADE: Reforma del sector privado debe incluir a las Mype.

- Diario Gestión (2008, noviembre 11). Sección Opinión (editorial). Las Mype aguardan el apoyo del MEF. Lima.

- Diario Gestión (2008, noviembre 25). Sección Economía. Podrán ingresar 72 productos textiles de China sin pagar aranceles. Lima.

- Flores Soria, J. (2005). Mypes. Lima: Centro de Especialización en Contabilidad y Finanzas.

- Gálvez León, L. (compilador) (2000).Desarrollo económico local y microempresa. Lima: Instituto de Desarrollo Urbano.

- Gonzales, J. (2001).Redes de informalidad en Gamarra. Lima: Universidad Ricardo Palma, Editorial Universitaria.

- Gonzales Vigil, F. (Editor) (2006). Tópicos de negociaciones comerciales internacionales: metodologías y aplicaciones relevantes para el Perú. Lima: Centro de Investigación de la Universidad del Pacífico. 
- Hermoza Ríos, J. (2000).Pequeña empresa en América Latina, alternativas para el desarrollo. Ediciones del Congreso del Perú.

- Irigoyen, H.(1998).Pymes, su economía y organización. Buenos Aires: Ediciones Macchi.

- Portocarrero Maisch, F. (1999). Microfinanzas en el Perú. Lima: Universidad del Pacífico, Centro de Investigación.

- Pressperú (2008, 17 de octubre). Ministra Mercedes Araoz inauguró programa Gamarra Exporta. www.pressperú.com

- Programa de Mejoramiento de la Competitividad para Pymes (ProCip) (2001). La clave del comercio: libro de respuestas para los pequeños y medianos exportadores (versión peruana). Lima: Centro de Comercio Internacional UNCTAD/OMC (CCI). Lima.

- Proinversión (2006). MYPEqueña empresa crece: Guía para el desarrollo de la micro y pequeña empresa. Lima: Proinversión.

- Ruiz Caro, A. (2007).Riesgos del TLC Perú-EEUU. Estudio auspiciado por OXFAM. Lima.

- Trejos Solórzano, J. (2003).La microempresa en el Perú a inicios del siglo XXI: magnitud, importancia y características. Serie: Desarrollo Económico Local, No 1.Lima: Promoción del Desarrollo Sostenible.

- Vereda del Abril, A. (2001).Emprendedores, microempresas y microcréditos, una propuesta para el desarrollo del Perú. Madrid: Fundación Iberoamericana para el Desarrollo.

- Villarán, F. (Coordinador) (2001). Competencias necesarias para la creación y gestión exitosa de pequeñas y microempresas en el Perú. Lima: Ministerio de Educación. Dentro del Programa Especial Mejoramiento de la Calidad de la Educación Peruana. En: www. mintra.gob.pe

- Villarán, F. (1998). Riqueza popular: pasión y gloria de la pequeña empresa. Artículo titulado: "Soledades que matan". Lima: Editorial del Congreso del Perú.

- Villarán, F. (1998). Riqueza popular: pasión y gloria de la pequeña empresa. Artículo titulado: "La maldición del modelo económico". Lima: Editorial del Congreso del Perú.

- Villarán, F. (1998).Riqueza popular: pasión y gloria de la pequeña empresa. Artículo titulado: "Alianza para la guerra". Lima: Editorial del Congreso del Perú. 